\title{
La Economía Solidaria en la politización del trabajo escondido de las mujeres
}

\author{
Beatriz Eugenia Cid Aguayo' (iD 0000-0003-0105-3553 \\ Loreto Patricia Arias Ramírez' (iD 0000-0003-2941-2343 \\ 'Universidad de Concepción, Departamento de Sociología, Concepción, Chile. \\ http://www.udec.cl/pexterno/
}

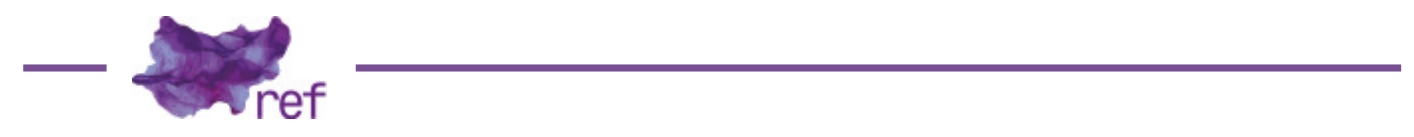

Resumen: ¿Existen redes económicas solidarias en los asentamientos urbanos irregulares contemporáneos? ¿Es posible potenciarlas? Este trabajo se basa en un proceso de Investigación Acción sobre redes de colaboración económica en 'campamentos', ubicados en la ciudad de Talcahuano, del Gran Concepción; una zona desindustrializada y neo-extractivista de Chile. La investigación, que se realizó en colaboración con una fundación tradicional, consistió de un conjunto de talleres participativos desarrollados con mujeres. Sus hallazgos permiten visibilizar y reconocerle colectivamente la heterogeneidad de las prácticas económicas realizadas por las mujeres en torno a la economía de los cuidados y la reproducción de la vida. Como tal, permiten un debate acerca de la relación entre los campos de la economía feminista y la economía solidaria.

Palabras clave: trabajo doméstico, economía feminista, economía solidaria, género, campamentos

Economia Solidária na politização do trabalho oculto das mulheres

Resumo: Existem redes econômicas solidárias nos assentamentos urbanos contemporâneos e irregulares? É possível reforçá-las? O trabalho apresentado baseia-se num processo de Pesquisa Ação, sobre as redes de colaboração econômica em favelas situadas na cidade de Talcahuano, Grande Concepción; Zona desindustrializada e neo-extractivista do Chile. A pesquisa foi desenvolvida em parceria com uma fundação tradicional, e consistiu num conjunto de oficinas participativas feitas com mulheres. Suas descobertas permitem visibilizar e reconhecer coletivamente a heterogeneidade das práticas econômicas realizadas pelas mulheres em torno à economia dos cuidados e a reprodução da vida. Isto permite o debate sobre a relação entre a economia feminista e a economia solidária. Palavras-Chave: Trabalho doméstico; Economia feminista; Economia Solidária; género; Favela.

Solidary Care Economy in the Politization and Socialization of Women's Hidden Labour Abstract: Are there economic solidarity networks in contemporary irregular urban settlements? Is it possible to boost them? This work is based on an Action Research process on economic collaboration networks in "campamentos" (shantytowns), located in the city of Talcahuano, Gran Concepción, a deindustrialized and neo-extractivist zone of Chile. The search, carried out in collaboration with a traditional foundation, consisted of a set of participatory workshops developed with women. The findings permit to collectively recognize and acknowledge the heterogeneity of economic practices carried out by women around the economy of care and reproduction of life. As such, it allows a debate about the relationship between the fields of feminist economics and solidarity economy.

Key words: Domestic Labour, Feminist Economics, Solidarity Economy, Gender, Shantytowns.

\section{Introducción}

Este trabajo se basa en un proceso de Investigación Acción sobre redes de colaboración económica en asentamientos urbanos irregulares, 'campamentos', ubicados en la ciudad de Talcahuano, del Gran Concepción, una zona desindustrializada y neo-extractivista del Chile contemporáneo. La investigación se realizó en colaboración con una fundación tradicional que trabaja en la regularización de asentamientos urbanos, y que en particular le interesaba explorar 
las potencialidades y la viabilidad de las estrategias de la economía solidaria para mejorar su enfoque de intervención en un contexto que se percibía fuertemente individualista. El estudio se organizó a través de un conjunto de talleres desarrollados con mujeres en tres 'campamentos', utilizando una versión culturalmente contextualizada de las metodologías propuestas por el enfoque de Katherine Gibson-Julie Graham (2006) acerca de economía comunitaria. Durante estos talleres se problematizaron la variedad y densidad de las prácticas económicas locales para proyectar imaginaciones económicas alternativas. El desarrollo y los resultados de esos talleres cuestionan la percepción social acerca de la preminencia de un comportamiento individualista y permiten un debate sobre la posible relación entre dos cuerpos literarios y políticos: la economía solidaria y la economía feminista de los cuidados. La discusión acerca de la economía de los cuidados reconoce el valor económico del conjunto de actividades desarrolladas para cuidar a otras personas -especialmente dependientes como niños, ancianos y enfermos- y más en general reproducir la vida en forma amplia. Los cuidados se reparten entre las familias, servicios del Estado, el sector privado y organizaciones comunitarias. Sin embargo, en nuestra sociedad, una parte importante de la misma se lleva a cabo al interior de los hogares, en forma no remunerada e invisibilizada, por las mujeres. Los esfuerzos para medir el trabajo doméstico de los cuidados, a través de encuestas de uso del tiempo, muestran que éste representa hasta el $40 \%$ del PIB. En un sentido más político y analítico, se puede argumentar que la economía del cuidado produce el tejido real de la vida; y como tal es estructuralmente, anterior, y una condición previa para la economía formal. Asimismo, muchas veces se organiza a través de valores no capitalistas tales como la cooperación y los afectos. El proyecto teórico y políico de la economía solidaria, a su vez, se ha propuesto hacer visibles y promover formas de producción, de intercambio y de consumo que arraigan el comportamiento económico en un marco ético y político. En otras palabras, es una forma heterogénea de economía la que prioriza el trabajo y la comunidad sobre la lógica de acumulación de capital. Este marco incluye desde sectores clásicos de la economía social, como asociaciones comerciales, cooperativas, empresas sociales, etc., a diversas expresiones de la economía popular, como el trueque y los sistemas de ahorro colectivo.

El proyecto de economía solidaria, en su agenda de visibilidad y articulación de otras economías, ha hecho un poco esfuerzo sistemático por reconocer la densidad económica del cuidado en los hogares. Esto último resulta paradójico ya que, a pesar de que las labores domésticas son ciertamente traspasadas por la dinámica de poder del patriarcado, éstas también representan un área de la economía organizada no por autointerés, sino por cooperación y afecto. Como consecuencia de esta baja visibilidad, no ha habido un esfuerzo sistemático para politizar y desarrollar sistemas cooperativos para reorganizar el trabajo de cuidado. Creemos que una discusión de la economía del cuidado a través de los lentes de la economía solidaria puede enriquecer ambos. Ello pues abre nuevas posibilidades políticas y económicas al feminismo -en términos de hacer públicas, valiosas y políticas las actividades domésticas- y a los movimientos de la economía social, ya que abre otro espacio económico para su discusión política.

El estudio se desarrolló con elementos de un enfoque Investigación Acción Participativa en tres 'campamentos' de los cerros de Talcahuano, ciudad de Concepción, la Región Biobío. El análisis social e histórico de los 'Campamentos' en Chile nos dice que su tratamiento tiene cambios con el tiempo. En los años 80, en el contexto de la dictadura, fueron vistos como lugares que fomentaban la organización activa y la economía solidaria. En el contexto democrático neoliberal, en el que los procesos de erradicación son una tendencia importante de una política de vivienda pública, se los considera como un terreno de clientelismo político y de comportamiento individualista, de escaso arraigo territorial, debido a la precariedad y temporalidad de los asentamientos. El estudio mostró diferencias importantes entre los 'campamentos' estudiados, en los que uno de ellos era ciertamente individualista y dependiente de redes clientelistas, pero los otros dos mantenían importantes y activas redes económicas solidarias, desafiando así la percepción que la literatura tiene acerca de la pérdida de lazos solidarios. Más importante aún, esos espacios de creatividad económica y asociatividad fueron reunidos casi exclusivamente por mujeres que, a través de la cooperación, buscan mejorar, aliviar y organizar su trabajo de cuidado familiar. Como resultado, construyen redes fluidas de ahorro colectivo, seguros y atención infantil que socializan su organización de economía doméstica. A pesar de esto, las mujeres todavía no reconocen esas acciones como actividades económicas valiosas, ya que no son vistas como parte de la economía monetaria. Estos resultados permiten plantear la cuestión de si la promoción popular de la economía solidaria en contextos vulnerables, además de promover procesos económicos productivos formales (como el modelo cooperativista), debe realmente comenzar por politizar, socializar y valorar aquellas actividades desarrolladas de manera invisible, gratuita y generosa por las mujeres.

\section{Algunos elementos de contexto}

El gran Concepción es el tercer conurbano más grande de Chile. Su crecimiento y el de los sectores aledaños estuvo principalmente marcado por políticas industrializadoras de desarrollo 
tipo Industralización por Sustitución de Importaciones (ISI) en las primeras décadas del siglo XX (Pablo FUENTES; Leonel PÉREZ, 2012, p. 35). Como tal llegó a ser una ciudad bastante industrializada en los sectores de la minería, metalúrgica, textilería, zapatería, vidriería y cerámica, lo que se asoció a un importante desarrollo portuario. Como tal, fue una importante atractora de mano de obra. Parte importante de dicha población fue asentada en barrios y poblaciones construidas por las mismas grandes empresas bajo políticas de paternalismo industrial. Sin embargo, también se extendió la vivienda precaria, cuyo primer espacio fueron conventillos y asentamientos irregulares. Desde el ciclo neoliberal instalado por la dictadura militar y mantenido en los gobiernos posteriores, mucho de ese desarrollo industrial ha entrado en un ciclo de declive, que ha puesto a los habitantes y barrios de la región en una creciente situación de vulnerabilidad económica.

Solo en la región del Biobío existen 152 campamentos que albergan a 8.088 familias. El Biobío es la segunda región con más cantidad de campamentos en Chile, solo antecedida por la Región Metropolitana. Las cifras arrojadas por la última Encuesta Nacional indican que el número de familias se ha acrecentado. El $59 \%$ de la población de campamentos en la octava región es menor de 30 años y corresponde mayoritariamente a mujeres. El principal motivo para irse a vivir a un campamento es provocado por la imposibilidad de tener un lugar donde vivir $(28,9 \%)$, seguido por la compleja situación económica y laboral de las familias $(15,3 \%)$. Este motivo se relaciona con el imaginario en torno al anhelo de "la casa propia" (14,5\%). Cabe destacar que un $8,6 \%$ de los hogares menciona la catástrofe del terremoto del 2010 como un motivo para vivir en campamentos (FUNDACIÓN TECHO-CHILE, 2015).

El sector de Talcahuano, donde se localizan los campamentos estudiados, tiene como principal actividad productiva la pesquería industrial y artesanal, como también la actividad portuaria. De esta manera, los trabajos que realizan los habitantes de los campamentos tienen que ver principalmente con dichas actividades. Solo en la bahía de San Vicente se encuentran al menos 20 campamentos ubicados en los cerros. Estos últimos constituyen la periferia de la comuna de Talcahuano y se sitúan en lugares no urbanizados.

La historia de los campamentos ha ido variando con respecto a los procesos políticos económicos en los que se insertan. La literatura describió a los campamentos como sectores marginados, en los cuáles la sobrevivencia cotidiana se resolvía a través de extensas redes de solidaridad y compadrazgo (Larissa ADLER, 1989); un espacio de movilización política -el movimiento de pobladores- (Vicente ESPINOZA, 1988) y también con una amplia gama de estrategias solidarias (Luis RAZETO, 1987). En textos más contemporáneos -con un sistema de mercado consolidado- se evalúa que es difícil encontrar estas prácticas cuando el modelo económico promueve la competencia y el individualismo. Liza Mónica Ayuso (2006) describe las barriadas argentinas, habla de violencia estructural, estatal e interpersonal, donde el neoliberalismo ha quebrado el tejido social del territorio; Donna M. Goldstein (2013) observa la violencia sexual, racial y de clase en las favelas brasileñas. Manuel Garretón (2010) describe el rol del Estado en promover el clientelismo y la dependencia subsidiaria. El Estado subsidiario se ha caracterizado por salvaguardar el consumo, es decir, mantener la demanda corrigiendo los problemas de exclusión asociados al modelo a través de diversas formas de asistencia, es decir, a través de estrategias y acciones focalizadas. Las políticas subsidiarias habrían apuntado a compensar las manifestaciones de dicha pobreza y exclusión pero no a erradicar sus causas. En este contexto, las acciones anteriores no solamente influyen en términos materiales, sino también simbólicos, construyendo una cultura de la dependencia dentro de los sectores no privilegiados, los cuales muchas veces esperan recibir subvenciones, sin tampoco prefigurar un objetivo de transformación a largo plazo. Este paternalismo es auspiciado por el Estado (GARRETÓN, 2010), promovido por el discurso de la izquierda y ocupado por el populismo de la derecha. En suma, la construcción analítica que se hace de los campamentos ha variado fuertemente, lo que lleva a la pregunta política de si la solidaridad es un valor del pasado, ya totalmente perdido; o si, por otra parte, existen bases reales para pensar en una estrategia basada en la economía solidaria.

\section{Economía solidaria y economía de los cuidados: posibles proximidades}

El concepto de economía solidaria se corresponde tanto a un proyecto político como un desafío analítico conceptual; y más aún, se presenta como una apuesta de redefinición epistémica respecto de los supuestos del análisis económico. Ambas dimensiones no son disociables, pues un proyecto político necesita crear un lenguaje teórico y epistémico que lo sostenga, pero metodológicamente pueden ser pensados como abordajes diferentes. En términos políticos, busca construir una economía con fundamentos éticos solidarios centrada en la valorización del proceso de trabajo y la cooperación (RAZETO, 1987, 1993,1997), con "una orientación fuertemente crítica y decididamente transformadora respecto de las grandes estructuras y los modos de organización y de acción que caracterizan la economía contemporánea" (RAZETO, 1997 p. 4). En términos 
analíticos se ha buscado un espacio teórico desde el cual comprender e imaginar formas alternativas del actuar económico, lo que implica también una respecto a las definiciones antropológicas del comportamiento económico (Anup DASH, 2014).

Para este proyecto, el trabajo clásico de Karl Polanyi representa un importante punto de partida. En particular, el autor redefine el análisis económico desde un ejercicio deductivo formal de modelación a partir de supuestos predefinidos -tales como la escasez, la acción racional y la maximización de utilidad- a un ejercicio de análisis empírico sustantivo de las prácticas históricas de movilización, y organización de recursos para resolver las necesidades humanas. Dichas prácticas no corresponden a una esfera autónoma y autorregulada, sino que están arraigadas en la vida social e institucional de una comunidad. De esta manera, la economía es inseparable de la ética, la cultura y la política.

Desde la categoría de arraigo ha habido una reelaboración de los supuestos clásicos de la ciencia económica. Las categorías de deseo y necesidad dejan de ser vistas como ilimitadas, universales y connaturales al ser humano, y pasan a verse como procesos socialmente construidos. Esto es, provenientes de instituciones sociales y constructos culturales que promueven el consumo ilimitado. La afirmación de la historicidad y contingencia de las necesidades tiene como resultado la relativización del supuesto de escasez. Así, la redefinición de estos supuestos económicos permite la posibilidad de pensar y construir otras economías, donde los valores y normas sociales instituyan otras relaciones económicas que prioricen la producción y reproducción intergeneracional de la vida.

En una vertiente más contemporánea y de raíz postestructuralista, el trabajo de GibsonGraham (2008) releva la heterogeneidad y proliferación de prácticas económicas empíricas que han sido invisibilizadas por el discurso económico científico formal-de la ciencia económica- y por el lenguaje e imaginario económicos convencionales. Ambos hegemonizados por lo que las autoras llaman un discurso capitalocéntrico, esto es un discurso que tiende a identificar sociedad con economía y economía con capitalismo. Su trabajo apunta a mostrar que el empleo asalariado, la empresa de capital y el mercado capitalista corresponden a un ámbito del universo económico posible, y que tras ellos subyacen una diversidad de relaciones económicas -no capitalistas e incluso no monetarias- que no logran ser comprendidas desde el lenguaje económico convencional y que, de hecho, pueblan una buena parte del universo económico, siendo claves para la reproducción de la vida y la mantención del bienestar. La superación del discurso capitalocentrista -o como Polanyi diría en 1957, la recuperación de un análisis económico sustantivo- es una condición de posibilidad para abrir la imaginación económica. Solo es posible un proyecto político de construcción de otras relaciones económicas a partir de la generación de un lenguaje capaz de dar cuenta de otras relaciones económicas posibles.

En términos de movimiento político económico, la economía solidaria alberga colectivos que promueven diversidad de relaciones económicas incluyendo intercambio, de donación, de reciprocidad, de comensalidad y de cooperación que adoptan múltiples formas, desde compras a través de dinero, trueques, clubes, talleres laborales, cooperativas y empresas comunes. Más allá de la forma económica que se adopte, la importancia radica en los fundamentos éticos solidarios a los que se suscriban. Estos apuntan -en sus vertientes más políticas-a la "emancipación de las fuerzas productivas", es decir, la reunificación del trabajo y los medios de producción; trabajo intelectual y trabajo manual, bienes económicos y necesidades humanas, gestión democrática y prácticas innovadoras de organización del trabajo (Euclides MANCE, 2000). El debate se extiende hacia la problematización de la división internacional del trabajo por medio del derecho de los pueblos a su propio desarrollo económico, cultural, social y humano, así como por el cuestionamiento a los términos de intercambio vigentes y por el comercio justo.

La economía feminista, por su parte, ha hecho un amplio ejercicio de develar los sesgos androcéntricos que subyacen en la economía como disciplina, y que en algunos sentidos se extiende al pensar de la economía solidaria. Este androcentrismo se expresa en que solo se reconoce como económico esferas históricamente masculinizadas-asociadas al trabajo público, productivo y crematístico-, mientras que esferas, roles, espacios históricamente femeninos -lo doméstico, el cuidado, el oikos- son definidas como lo no-económico. Estos binarismos productivo/ reproductivo, mercado/no mercado, autointerés/altruismo no son simple dicotomías, sino expresan un ordenamiento jerárquico genderizado entre lo racional y lo irracional (DASH, 2014). Lourdes Benería (1999), por su parte, señala que desde el homo oeconomicus hasta el hombre de Davos la ciencia económica ha ignorado los vínculos afectivos y las relaciones de obligaciones y cuidado que vinculan a las personas en contextos familiares y doméstico. De esta manera, el trabajador libre, el actor económico racional, maximizador y autointeresado, no está genéricamente construido, ni tampoco da cuenta de actores económicos reales que están inscritos -arraigados- en entramados institucionales y afectivos.

Una de las contribuciones centrales del pensamiento feminista es la ampliación del concepto de trabajo -como actividad humana creadora y relacional- más allá de los límites del trabajo 
asalariado y productivo. En la discusión feminista del concepto de trabajo se vienen a visibilizar dos grupos de actividades que amplían no solo el concepto de trabajo, sino también el de trabajo doméstico (Cristina CARRASCO, 1999). De esta manera, se diferencian, por un lado, la gestión y producción de los bienes domésticos, y por otro lado, las tareas de "cuidado" y "apoyo" a personas dependientes, más adelante llamado trabajo de cuidado (Cristina BORDERÍAS; CARRASCO, 1994). Así, trabajo doméstico es el conjunto de trabajos realizados al interior del espacio doméstico. Es productivo y reproductivo, invisible, no remunerado, y no está protegido, ni regulado. El trabajo de cuidados, por su parte, es el conjunto de actividades que se realizan orientadas a cuidar a los más vulnerables, como los niños, las personas enfermas y de tercera edad, como también aquella población dependiente, incluyendo a hombres adultos (Gisella BOCK; Pat THANE, 1996). La organización de los cuidados no es exclusivo del hogar, se puede realizar desde el ámbito privado (clínicas, hospitales), público (asistencias sociales del Estado), e incluso desde el voluntariado de organizaciones comunitarias (Shahra RAZAVI, 2007). Por lo tanto, fuera de lo doméstico, este trabajo es reconocido y valorado. En contextos de Estados reducidos, gran parte de las actividades son absorbidas al interior de los hogares por las mujeres, sin reconocimiento.

Si bien tanto el trabajo doméstico como el trabajo de cuidados coinciden en la esfera del hogar, el trabajo de cuidados puede proyectarse en el mercado y convertirse en un servicio o mercancía. Es decir, deja de ser trabajo invisible, pero solo como proyección que se extiende hasta donde se pueda pagar o mantener, de lo contrario es absorbido por mujeres para desarrollarse nuevamente en conjunto y bajo la dimensión del trabajo doméstico. De esta manera, hablar ya del trabajo de cuidados nos lleva a analizar las redes que se cristalizan para resolver la organización de los cuidados en donde se encuentran cuatro actores involucrados: el Estado, la familia, las organizaciones comunitarias y las empresas privadas (RAZAVI, 2007). En este sentido, podemos decir que la organización del cuidado se da través de encadenamientos múltiples y no lineales que traspasan las fronteras de la esfera doméstica. (Corina RODRÍGUEZ ENRÍQUEZ; Laura PAUTASSI, 2014). Si bien algunos de estos trabajos se desarrollan en ámbitos institucionalizados del sector público y privado, esta ampliación nos remite a repensar las nociones de pleno empleo, calificación, y aun de trabajo emancipado, ya que no hay trabajo emancipado para las mujeres sin la división de las tareas domésticas entre todos los que conviven en un mismo espacio (Miriam NOBRE, 2015).

Ahora bien, al reconocer el trabajo doméstico y de los cuidados como un ámbito económico real, tenemos también que reconocer que este es un tipo de trabajo que muchas veces se organiza por una racionalidad sustantiva, no monetaria y desmercantilizada (DASH, 2014). Sin embargo la "invisibilidad" que ha llegado a tener la economía de los cuidados -cuando es desarrollada en el ámbito doméstico y comunitario- se debe precisamente a que las actividades y flujos que no pasan por el mercado de intercambios no tienen expresiones monetarias -crematísticas-, de allí también la dificultad que existe para apreciar su magnitud y cuantificarlo.

En consecuencia de lo anterior, muchos de los grupos que habitan el universo de la economía solidaria cuentan con una fuerte presencia de mujeres. Ello es especialmente claro en relación con los llamados servicios de proximidad, donde se asumen los servicios sociales ante el achicamiento del Estado, en temas tales como agentes de salud, de promoción de la seguridad alimentaria y guarderías, con contratos y condiciones de trabajo precarias. Nobre (2015) señala la paradoja de que mientras funciones centrales para la reproducción social son desvalorizadas y mantenidas desde el sobre trabajo de las mujeres, la economía solidaria no se posiciona frente al tema, haciéndose parte del sesgo androcéntrico del pensar económico. Más aun, muchas de las organizaciones de la ES reproducen acríticamente dicha división social del trabajo, tanto al interior de la organización como afuera, permitiendo la doble jornada femenina. Así también, las organizaciones y la participación femeninas están escasamente visibilizadas en las estructuras de representación formal de la Economía Solidaria (NOBRE, 2015).

La organización doméstica y comunitaria de los cuidados es especialmente relevante en el contexto de reducción neoliberal del Estado, donde la precariedad y la inseguridad social hacen más relevante la economía de los cuidados, tanto al interior del hogar (cuidado de niños, ancianos y enfermos), como al exterior de éste. Estos cuidados dotan de recursos materiales (salud, vivienda), pero también simbólicos (las confianzas, poder contar siempre con...). De esta manera, es claro como ambos cuerpos teóricos -economía feminista y economía solidaria- enfatizan la cuestión de las necesidades humanas versus la utilidad o lucro. El análisis económico feminista ha observado la organización del trabajo familiar en relación a la satisfacción colectiva de necesidades, mientras las premisas de la economía solidaria llevan esta pregunta a escala comunal y societal, donde el trabajo se reparte a través de las jerarquías sociales y del género (Christine BAUHARDT, 2014). Sin embargo, es muy importante no olvidar que la economía doméstica es un espacio de relaciones humanas fuertemente contradictorias. Por una parte, se da por sentado la presencia de solidaridad, afectos, convivialidad, integración, cooperación en el trabajo y la 
comunidad en el consumo de los bienes y servicios. Por la otra, es también espacio de dominación patriarcal y heteronormativa. En este sentido, varias de las reivindicaciones feministas (Amaia PÉREZ OROZCO, 2014) constituyen una reacción contra distorsiones de la familia, por lo que es esperable que al menos en parte puedan encontrar adecuada satisfacción en el marco de la economía de solidaridad y específicamente en la ampliación y recuperación del contenido económico de la familia (RAZETO, 1993).

\section{Elementos de Método}

La pregunta que organizó este ejercicio de investigación se inspiró en el trabajo de GibsonGraham (2006), que busca visibilizar las prácticas reales de heterogeneidad y densidad económica territorial, tanto para los ojos del investigador como para las propias participantes del proceso de investigación. En este sentido, el objetivo del trabajo no es solo catalogar prácticas solidarias sumergidas en el contexto del Chile neoliberal y reconocer su dimensión de género, sino que estas prácticas sean reconocidas y valoradas por sus propias protagonistas, con miras a pensar la posibilidad de lo solidario como código y estrategia de relación económica. Es por ello que la investigación se organiza desde los códigos de Investigación Acción Participativa, pues su objetivo no es solo conocer un proceso social sino transformarlo, especialmente desde la subjetividad de sus protagonistas.

La Investigación Acción Participativa busca asegurar la producción colectiva del conocimiento, incluyendo las voces y discursos que no son usualmente escuchados, y por ende busca visibilizar temáticas que las investigaciones con metodologías tradicionales pueden pasar por alto. Una de sus las características "que lo diferencia de todos los demás, es la forma colectiva en que se produce el conocimiento, y la colectivización de ese conocimiento" (Orlando FALS BORDA; Carlos RODRÍGUEZ BRANDAO, 1987, p. 18). De esta manera, no interesan tanto los sentidos individuales como los sentidos colectivos que se les dan a las prácticas colectivas (Stephen KEMMIS; Robin MCTAGGART, 2007). Así también en la actividad investigativa no se borra a las investigadoras, sino que más bien se aúna a la producción de conocimiento, situando a las personas de campamentos como sujetos y no como objetos (Joel MARTí, 2002). En particular esta investigación se desarrolló en torno a una serie de tres series talleres desarrollados en campamentos del sector de los cerros de Talcahuano. Estos talleres se convocaron abiertamente a través de las organizaciones y dirigencias locales. Las asistentes fueron mujeres, todas madres, casadas y solteras. El rango de edad fue amplio, desde 17 hasta 60 años.

Los talleres se estructuraron a partir de una adaptación contextual de la metodología 'Assets Bassed Communities Development' utilizada por Gibson-Graham (2006), en el valle de Latrobe. El primer taller de la serie buscó iniciar una discusión sobre el lenguaje y forma de mirar de la economía solidaria. Los otros dos talleres apuntaron a la identificación de necesidades e intereses de la comunidad como también a las capacidades personales, colectivas y territoriales para abordar estas necesidades. Esto es, en el lenguaje de Gibson-Graham, el cultivo de sujetos capaces de reconocer las posibilidades de sus economías comunitarias. Cada taller está organizado para promover la participación y ser una experiencia amena de discusión dentro de la comunidad (KEMMIS; MCTAGGART, 2007; Jarg BERGOLD; Stefan THOMAS, 2012).

El registro se hizo a través cámaras fotográficas y audiovisuales y también por grabadora de voz. El análisis de los talleres transitó por la observación participante a través de notas de campo y el análisis del registro visual y de audio generado en los talleres.

\section{Prácticas económicas comunitarias en campamentos de los cerros de Talcahuano}

Como se señaló previamente, esta investigación fue parte de un proceso por el cual una fundación tradicional en Chile busca explorar las metodologías de la economía solidaria para mejorar su trabajo en campamentos. Para ello, se buscó reconocer y visibilizar colectivamente la heterogeneidad de las prácticas económicas desarrolladas localmente, esto es, el conjunto de actividades solidarias que llevan a cabo las personas para satisfacer sus necesidades y entrever las potencialidades de una economía solidaria de base comunitaria.

En primera instancia, lo que buscó esta investigación-acción participativa fue visibilizar participativamente 'las otras prácticas'. Aquellas actividades que existen bajo las sombras de un régimen económico neoliberal que ha sido, discursiva y materialmente, hegemónico. En este sentido pudimos recuperar cuatro tipos de prácticas cuyo contenido fue bastante diverso, respondiendo a necesidades diversas. Todas las actividades solidarias eran comúnmente en los campamentos conocidas por beneficios. Palabra de amplio uso y de semántica bastante interesante: aquello de lo que todos nos beneficiamos. Esto es, cualquier tipo de actividad colectiva que pueda generar ingresos para uso común o para alguna familia pasando por una necesidad especial. 
Las organizaciones de cooperación que se encontraron en los campamentos giraban en torno a cuatro necesidades:

La primera, de mayor uso y sistematicidad, constituye un sistema de cooperación en caso de tragedias. Este es el conjunto de estrategias realizadas con el objetivo de juntar bienes y dinero para alguna familia que se encuentra pasando por un período de crisis o tragedia familiar. Ello es especialmente relevante en el contexto de vulnerabilidad que representa la vida en campamentos. Por ejemplo, las casas son en su mayoría de material ligero y las familias se conectan ilegalmente -'se cuelgan'- al tendido eléctrico, por lo cual son muy recurrentes los incendios con pérdidas totales. De esta manera, las familias de los tres campamentos estudiados se han organizado para hacer frente a este tipo de situación. La responsabilidad de su organización recae generalmente en las dirigentas que organizan eventos que sirven para recaudar dinero, como 'mateadas' o bingos. Dichos eventos funcionan primero a través de la donación de insumos por parte de los vecinos, como también la activa participación de los vecinos como consumidores en la actividad. A partir de ello se logran generar relevantes montos de dinero a la familia afectada, y se refuerzan los, a veces débiles, lazos comunitarios.

Por ejemplo, cada vecina se compromete con hacer tal cantidad de calzones rotos, completos, sopaipillas, otras con navegaos y así (...) Antes cada vecina se compromete con la compra de tres completos, ponte tu (...) Así no nos sobra. (Pobladora, 2015)

De esta manera, se aseguran de no tener pérdidas, fortalecer lazos comunitarios y generar dinero para la familia afectada. Este tipo de ejercicio es importante por su difusión y recurrencia, es un contexto donde los sistemas de seguros están fuertemente privatizados. Por lo que este tipo de estrategias representa un apoyo importante, precisamente en el momento en que la reproducción de la vida y los cuidados están en crisis para el grupo familiar. Así también es relevante la organización de trabajo y recursos colaborativos que benefician no solo a la comunidad, sino una familia o persona más afectada por una tragedia. En este sentido, es un ejercicio de empatía importante, que demanda tiempo y trabajo por parte de las involucradas. Asimismo, refuerza las relaciones existentes de esa familia con el resto de la comunidad.

Menos recurrente que el anterior, pero documentado en dos de los campamentos son el sistema de ahorro en conjunto, con objetivos específicos. En uno de los campamentos estudiados, existe una organización creada por pobladoras Ilamado 'El Taller', cuyo único objetivo es generar dinero a través de diversos beneficios y ahorrar dinero en forma sistemática por medio de cuotas para un pozo común:

Beneficio quiere decir que hacemos venta de completo, venta de plato de comida puede ser papas mayo con pollo arvejado, con chuletas (...) A la gente de afuera, ofrecemos, cada socia se hace cargo de vender, ponte tú, diez platos. Entonces hacemos a dos lucas, si hacemos cien platos son doscientos mil pesos. Puedes gastar 80 lucas en gasto y te ganas ciento veinte, una cosa así. (Dirigenta del campamento Coliumo Bajo, 2015)

Esta organización compuesta únicamente por mujeres de los campamentos se reúne semanalmente en la sede vecinal, con el propósito de diseñar actividades en conjunto para generar más ahorro colectivo. El propósito del ahorro, en este caso, es financiar instancias recreativas asociadas a las actividades familiares y el trabajo de los cuidados, esto es, fiestas infantiles para Navidad y el Día del Niño, compra de regalos navideños, celebración del Día de la Madre y compra de útiles escolares.

Así también existen grupos - menos organizados- de ahorro compartido, que buscan juntar dinero de manera colectiva todos los meses, sin necesariamente implementar actividades específicas para recaudar dinero.

Todos los meses voy poniendo 3 luquitas, con mi mejor amiga, hacemos un pozo y decimos ya..., no lo vamos a gastar hasta diciembre, ponte tú, y.... pasan los meses y... va creciendo el chanchito. (Señora de unos 40 años del campamento Rucamanque, 2015)

Esta práctica da cuenta de cómo el apoyo social y el esfuerzo colectivo aparecen relevantes incluso para promover la práctica privada del ahorro. Asociado a los sistemas de ahorro se documentan también sistemas de compras compartidas. Estas estrategias, similares a los históricos 'comprando juntos', apuntan a obtener mejores precios en productos mayoristas utilizando principalmente lo acumulado en el pozo común generado por el ahorro. Los bienes adquiridos corresponden en su totalidad a artículos relacionados con el desempeño de los cuidados familiares: regalos de navidad, canastas familiares con alimentos y bebestibles especiales para festividades, compra de listas de útiles escolares. No se documenta eso si la compra es de necesidades básicas como alimento o ropa. Es interesante también que este ejercicio envuelve la práctica democrática de juntarse y tomar acuerdos acerca de qué se va a comprar y los mecanismos para hacerlo.

Respecto a compras colectivas para el mejoramiento de infraestructura comunitaria, como es el caso del mantenimiento de sedes y otros espacios públicos, las organizaciones gestionan en 
forma colectiva y democrática los recursos transferidos desde la fundación de apoyo. Además de las prácticas anteriores, existe un conjunto de expresiones solidarias, que llamaremos encuentros, que tienen la característica de ser más pequeñas y fugaces, pero no por eso menos importantes para la vida de las familias de los campamentos. Un ejemplo de estos eventos, dotado de una fuerte carga simbólica, es la llamada rifa del huevo que acompaña muchos de los eventos sociales del campamento. Esta actividad consiste en que cada asistente colabora con uno o dos huevos, los que se juntan en docenas, que son posteriormente rifadas entre los asistentes, con venta de números de muy bajo costo, permitiendo generar un excedente monetario para uso colectivo de la organización

(...) se lleva dos huevos por persona y esos huevos se rifan. Se compran tres número a cien pesos, y bueno ahí se rifa y la que se ganó los huevos se los lleva, de siete, de a seis, de cinco, según los huevos que lleguen y todo los lunes (Dirigenta del campamento Las Algas, 2015).

Estas actividades, que involucran un bajo esfuerzo económico y de gestión de las participantes, permiten mantener una cultura y una práctica cotidiana de la cooperación que complementa y profundiza las actividades más complejas, construyendo espacios de encuentro entre las pobladoras y demostrando que lo económico y lo social suele fundirse:

Todo es para caja, todo es para caja. Y todo es para todas, no hay ninguna que vaya a recibir más que la otra. No, porque todas pagamos igual, y todas trabajamos iguales, todos pagan la misma cuota, todas venden la misma cantidad, así que es todo igual (Dirgienta campamento Las Algas, 2015).

La confianza juega un papel central en la construcción y mantención de estas prácticas. Como señala José Luis Coraggio (2007), para que la economía de la solidaridad se abra camino en las prácticas cotidianas, las negociaciones entre los vecinos deberían ir orientadas de tal manera que, cuando entren en contradicción el individuo con la comunidad, lo que prevalezca sean los intereses solidarios y el beneficio de la comunidad. Las mujeres y sus preocupaciones por la reproducción general de la subsistencia, cuidado y crianza protagonizan la actividad económica cooperativa de los campamentos. Son ellas las que organizan, participan y sostienen las instancias económicas y solidarias. Esto se ancla no en las peculiaridades de la subjetividad femenina, sino en la marcada división de los trabajos por roles de género. En los campamentos estudiados, son las mujeres quienes cargan el peso del trabajo doméstico y la responsabilidad por la reproducción biológica y social de sus familias; y es desde esos roles y responsabilidades que están presentes en la vida económica de su comunidad. Los hombres -cuándo están presentes, por su parte, entran y salen del territorio, y su vida económica transita por fuera del campamento. Este hecho permite reconocer que el trabajo de cuidados es central en las estrategias económicas de colaboración solidaria desarrolladas en los territorios. En ellos se viven prácticas de economía solidaria que complementan y enriquecen la economía de los cuidados.

\section{Los caminos de la visibilidad}

Las prácticas solidarias relevadas en este documento no son solo invisibles para los observadores externos a la vida de los campamentos, sino que también son escasamente reconocidas como tales por parte de las comunidades que las practica. Si bien son reconocidos como espacios de organización social y satisfacción de ciertas necesidades colectivas, no son conceptualizadas ni como trabajo, ni como actividades económicas. Al respecto es muy revelador un gesto realizado espontánea y sistemáticamente por las participantes en los primeros talleres: cada vez que se nombra una actividad que no genera ingresos monetarios directos, se levantan silbidos y pifias. Ello muestra muy claramente que, a pesar de la importancia evidente del trabajo reproductivo, doméstico y comunal desarrollado por las mujeres para reproducir la vida, finalmente estas prácticas no son validadas como trabajo verdadero y socialmente reconocido.

En los talleres se condujo explícitamente una reflexión sobre el valor de dichas actividades para la producción de bienestar y reproducción de la vida cotidiana. Esto es, reconocer el valor económico en prácticas que escasamente han sido conceptualizadas como tales. A partir de ahí, las mujeres participantes hicieron una explícita toma de conciencia del carácter productivo de su actividad familiar y comunitaria, definiéndola como un elemento central de la estructura de satisfacción de sus necesidades. Ello en sí mismo es valioso, pues contribuye al empoderamiento de las mujeres en contextos vulnerables, que es definido por la ONU como tener el poder y control sobre sus propias vidas, para lo cual se necesita desarrollar la autoestima, ampliar sus opciones, y no se refiere únicamente al poder dominante, o a formas de poder controlador, sino más bien a representaciones alternativas de poder: poder para, poder con, y poder desde el interior (ONU MUJER, 2018). De esta manera, ellas transforman la percepción de su trabajo cotidiano desde una construcción desde la desvalorización y la carencia a una del poder hacer, donde su trabajo es reconocido como central para la vida económica de sus familia y comunidades. En uno de los campamentos trabajados, este proceso dio un paso más allá. En particular, las mujeres 
participantes reconocieron ampliamente el carácter social, colectivo y solidario de su trabajo. Este reconocimiento generó en ellas también el deseo de potenciarlo y expandirlo. Y los últimos talleres fueron verdaderas explosiones de creatividad social, donde, desde visiones muy innovadoras, fueron imaginando y planificando futuros económicos ampliados. En este campamento, la observación de sus propias prácticas termina creando una nueva realidad económica: la posibilidad de que el trabajo asociado pueda institucionalizarse y contribuir a formas de alternativas para subsistir y crear autonomía. Este proceso, sin embargo compite con los sentimientos de desesperanza, dependencia y clientelismo que horadan procesos de construcción de autonomía.

\section{Reflexiones finales}

En los campamentos analizados, las conversaciones comunes no hablan de capitalismo, de mercado, de patriarcado, ni de feminismo, menos se habla de combatirlo o modificarlo. Las estrategias desplegadas no pretenden tener relevancia política al respecto. Pese a ello, hasta cierto punto, mitigan la dependencia de las comunidades e individuos con respecto al mercado y al estado subsidiario; esto especialmente en situaciones de emergencia cuando tanto mercado como Estado no responden a las necesidades. Estas prácticas son producto de sus circunstancias, que permiten pequeñas o grandes expresiones de solidaridad, dependiendo de las características de la comunidad, reforzando valores como la constancia, la responsabilidad y la reciprocidad entre los socios y las socias. El conjunto de estas prácticas pueden continuar siendo invisibilizadas, o menospreciadas desde una mirada estructural que arroja estas experiencias a los márgenes de la vida económica y social; o por el contrario, se las puede observar desde sus posibilidades para una sociología de las emergencias (Boaventura DE SOUSA SANTOS, 2009). Es desde esta opción epistemológica que elegimos mirarlas: enfocarnos en sus posibilidades políticas y económicas antes que cegar nuestra mirada con los lentes oscuros para enfocarnos en los límites.

Los campamentos dependen casi totalmente del trabajo asalariado y de la inserción en el mercado informal, y es justamente ahí donde buscan satisfacer sus necesidades. Dada esta condición, nos parece remarcable que realicen ejercicios de autonomía, como los que expresados en la compra y el ahorro en conjunto, aun cuando esas prácticas se inscriben principalmente y casi exclusivamente en la esfera del consumo. Ello porque, de una forma u otra, demuestran nuevamente que la creatividad social no se agota, solo se esconde y se adapta a los contextos. Siguiendo a Gibson-Graham (2006), se observa que el poder discursivo del capitalismo no hegemoniza todo, sino que en contextos adversos perviven y florecen prácticas de cuidado mutuo, solidaridad, y abordaje colectivo de las necesidades de reproducción de la vida.

Las mujeres se organizan en torno a ciertas necesidades orientadas principalmente a complementar el trabajo de cuidados, asegurar canastas familiares para todas las socias a fin de año, al igual que regalos para sus niños y para ellas mismas. Todos ellos asuntos generalmente relegados al ámbito privado. En este sentido, la resolución colectiva en el ámbito público de necesidades antes inscritas en el ámbito privado se constituye como una poderosa posibilidad y cruce entre la economía solidaria y la economía feminista.

Siguiendo esta línea, si abrimos los márgenes de la economía, ejemplos como los jardines infantiles populares ilustran de buena manera cómo la socialización del trabajo de cuidados se saca de la esfera privada para resolverse en la esfera pública de forma colaborativa. Las ollas comunes, otrora realizadas tanto en los cordones industriales como en el movimiento de pobladores, también eran llevadas a cabo principalmente por mujeres. En los casos estudiados, las estrategias desplegadas por las pobladoras iban en dirección a complementar su trabajo de cuidados de manera de resolver aquellas necesidades, fuera del ámbito privado de su casa y en colaboración con otras mujeres.

En este aspecto es necesario ampliar el concepto de economía solidaria, reconociendo la centralidad de las estrategias de las mujeres para el abordaje asociativo de economías solidarias del cuidado. Este tipo de estrategias, enmarcadas en roles femeninos históricos, abren posibilidades para el avance y reconocimiento de una nueva economía, donde el trabajo de cuidados sea socializado y compartido. Donde, así también, la transformación de la economía en torno a la sostenibilidad de la vida contribuye a la superación de la contradicción material y simbólica entre trabajo productivo y reproductivo.

\section{Referencias}

AYUSO QUIÑONES, Liza Mónica. “La Guerra Contra Las Drogas, La Guerra Contra Los Pobres: Aspectos Socioeconómicos de la Política Pública". Revista Jurídica de La Universidad de Puerto Rico, San Juan, Puerto Rico, v. 75. 2006.

BAUHARDT, Christine. "Solutions to the crisis? The Green New Deal, Degrowth, and the Solidarity Economy: Alternatives to the capitalist growth economy from an ecofeminist economics perspective". Ecological Economics, Berlin, v. 102, p. 60-68. 2014. 
BENERÍA, Lourdes. "Mercados globales, Género y el Hombre de Davos." Feminist Economics, Houston, V. 3, n. 5. 1999.

BERGOLD, Jarg; THOMAS, Stefan. "Participatory Research Methods: A Methodological Approach in Motion" [1 10 paragraphs]. Forum Qualitative Sozialforschung / Forum: Qualitative Social Research, Berlin, v. 13, n. 1, art. 30. 2012.

BOCK, Gisella; THANE, Pat. Maternity and Gender Policies. Women and the Rise of the European Welfare States, 1880s-1950s. Londres y Nueva York: Routledge. Traducción castellana en Maternidad y políticas de género. La mujer en los estados del bienestar europeos. Valencia: Cátedra, 1996.

BORDERÍAS, Cristina; CARRASCO, Cristina. Las mujeres y el trabajo: aproximaciones históricas, sociológicas y económicas. Las Mujeres y el trabajo: rupturas conceptuales. Barcelona: Icaria Editorial, 1994.

CARRASCO BENGOA, Cristina. Mujeres y economía: nuevas perspectivas para viejos y nuevos problemas. Barcelona: Icaria Editorial, 1999.

CORAGGIO, José Luis. "El papel de la Economía Social y Solidaria en la Estrategia de Inclusión SOcial". In: SEMINARIO INTERNACIONAL ECUADOR AL 2020. PENSANDO EN ALTERNATIVAS PARA EL DESARROLLO, 2007, Quito, Ecuador, SENPLADES y FLACSO. p. 10-13.

DE SOUSA SANTOS, Boaventura. Una epistemología del sur: la reinvención del conocimiento y la emancipación social. Buenos Aires: Siglo XXI, 2009.

DASH, Anup. "Toward an epistemological foundation for social and solidarity economy" . UNRISD Occasional Paper: Potential and Limits of Social and Solidarity Economy, n. 3. 2014.

ESPINOZA, Vicente. Para una historia de los pobres de la ciudad. Santiago de Chile: Editorial Sur, 1988.

FALS BORDA, Orlando; RODRÍGUEZ BRANDAO, Carlos. Investigación Participativa. Montevideo: La Banda Oriental, 1987.

FUENTES, Pablo; PEREZ, Leonel. "Formación del Concepción metropolitano a través de los grandes conjuntos residenciales: Aportaciones del urbanismo moderno." Concepción: Atenea, n. 505, p. 33-78. 2012.

FUNDACIÓN TECHO-CHILE. Resumen Ejecutivo de la Encuesta Nacional de Campamentos. Centro de Investigación Social, 2015.

GARRETÓN, Manuel. "La democracia incompleta en Chile: La realidad tras los rankings internacionales". Revista De Ciencia Política, Santiago de Chile, v. 30, n. 1, p. 115-148. 2010.

GIBSON, Katherine; GRAHAM, Julie. A Postcapitalist Politics. Minneapolis: University of Minnesota Press, 2006.

GIBSON, Katherine; GRAHAM, Julie. "Diverse economies: performative practices for "other worlds."” Progress in Human Geography, Chicago, v. 32, n. 5, p. 613-632. 2008.

GOLDSTEIN, Donna. Laughter out of place: Race, class, violence, and sexuality in a Rio shantytown. California: Univ. of California Press, 2013.

KEMMIS, Stephen; MCTAGGART, Robin. "Participatory Action Research: Communicative Action and the Public Sphere”. In: DENZIN, Norman; LINCOLN, Yvonna. (Eds.). Strategies of Qualitative Inquiry, Sage, Thousand Oaks, p. 271-330. 2007.

ADLER de Lomnitz, Larissa. "Mujeres, redes y economía informal”. In: La Participación de la Mujer en la Vida Nacional. México: Universidad Autónoma de México, 1989. p. 196-213.

MANCE, Euclides. "Uma introdução conceitual às filosofias de libertação." Revista LibertaçãoLiberación, IFiL, Curitiba, v. 1. 2000.

MARTí OLIVÉ, Joel. "La Investigación Acción Participativa - Estructura y Fases". In: MARTí, Joel; MONTAÑÉS SERRANO, Manuel; RODRÍGUEZ-VILLASANTE, Tomás (Coord.) La investigación Social Participativa. España: Editorial El Viejo Topo, 2002. p. 79-12. 
NOBRE, Miriam. "Economía Solidaria y Economía Feminista: Elementos para una agenda". Las mujeres en la construcción de la economía Solidaria y la Agroecología: textos para la acción feminista. Brasil: SOF Sempreviva Organização Feminista, 2015.

ONU MUJER. Glosario de Igualdad de Género, emanado desde Important Concepts Underlying Gender Mainstreaming de la Oficina de la Asesora Especial en Cuestiones de Género y Adelanto de la Mujer, ONU MUJER. 2001. Disponible en: https://trainingcentre.unwomen.org/mod/glossary/ view.php. Acceso el 01/09/2017.

PÉREZ OROZCO, Amaia. Subversión feminista de la economía. Aportes para un debate sobre el conflicto capital-vida. Madrid: Traficantes de Sueños, 2014.

POLANYI, Karl. La gran transformación. Los orígenes políticos y económicos de nuestro tiempo. México: Fondo de Cultura Económica, 1957.

RAZAVI, Shahra. The Political and Social Economy of Care in a Development Context: Conceptual Issues, Research Questions and Policy Options. Ginebra: Instituto de Investigaciones de las Naciones Unidas para el Desarrollo Social (unrisd), 2007.

RAZETO MIGLIARO, Luis. Las empresas alternativas. Santiago de Chile: Ediciones PET, 1987.

RAZETO MIGLIARO, Luis. Los caminos de la Solidaridad. Santiago de Chile: Ediciones Vivarium, 1993.

RAZETO MIGLIARO, Luis. "Factor C: la solidaridad convertida en fuerza productiva y en el factor económico". In: ORTIZ, Humberto; MUNOZ, Ismael (Eds.). Globalización de la solidaridad. Un reto para todos. Perú: Grupo Internacional Economía Solidaria (GES) - Centro de Estudios y Publicaciones (CEP), 1997.

RODRÍGUEZ ENRÍQUEZ, Corina; PAUTASSI, Laura. La organización social del cuidado de niños y niñas. Elementos para la construcción de una agenda de cuidados en Argentina. Buenos Aires: ELA / CIEPP / ADC, 2014.

Beatriz Eugenia Cid Aguayo (beatrizcid@gmail.com; beatrizcid@udec.cl) es socióloga Pontificia Universidad Católica de Chile, Magister Universidad de Chile, PhD Sociología York University. Profesora Asociada del Departamento de Sociología de la Universidad de Concepción. Directora del Proyecto Fondecyt Nol 160186 "Cartografías de Heterogeinedad Económica: Estudios de Casos". Trabaja temas de economías heterogéneas, bienes comunes y género.

Loreto Patricia Arias Ramírez (lariasr92@gmail.com; loretoarias@udec.cl) é socióloga Universidad de Concepción, Asistente en la facultad de Ciencias Sociales y departamento de Sociología de la Universidad de Concepción. Trabaja en temas de economías heterogéneas y feministas.

\section{COMO CITAR ESTE ARTíCULO DE ACUERDO CON LAS NORMAS DE LA REVISTA}

AGUAYO, Beatriz Eugenia Cid; RAMIREZ, Loreto Patricia Arias.. "La Economía Solidaria en la politización del trabajo escondido de las mujeres". Revista Estudos Feministas, Florianópolis, v. 27, n. 2, e54486, 2019.

\section{CONTRIBUCIÓN DE AUTORÍA}

Loreto Arias: Revisión de manuscritos, recogida y análisis de datos.

Beatriz Aguayo: Redacción y discusión de resultados.

FINANCIACIÓN:

"FONDECYT 1 160186: Cartografías de Heterogenidad Económica, los casos de la región del Biobío, Maule y Valparaíso". 
CONSENTIMENTO DE USO DE IMAGEM

No aplicable

APROVAÇÃO DE COMITÊ DE ÉTICA EM PESQUISA

No aplicable

CONFLITO DE INTERESSES

No aplicable

LICENÇA DE USO

Este artigo está licenciado sob a Licença Creative Commons CC-BY Internacional. Com essa licença você pode compartilhar, adaptar, criar para qualquer fim, desde que atribua a autoria da obra.

\section{HISTÓRICO}

Recebido em 11/12/2017

Reapresentado em 01/08/2018

Reapresentado em 12/09/2018

Aprovado em 23/1 1/2018 